\title{
Horizons/Théâtre
}

Revue d'études théâtrales

13 | 2018

Les arts du spectacle dans l'Afrique subsaharienne - 1

\section{Aux sources du théâtre ivoirien moderne : cas des premiers auteurs et leurs œuvres}

Florence Kouassi Akissi

\section{(2) OpenEdition}

1 Journals

Édition électronique

URL : https://journals.openedition.org/ht/1166

DOI : $10.4000 /$ ht. 1166

ISSN : 2678-5420

Éditeur

Presses universitaires de Bordeaux

\section{Édition imprimée}

Date de publication : 31 décembre 2018

Pagination : 108-117

ISBN : 979-10-300-0318-5

ISSN : 2261-4591

Référence électronique

Florence Kouassi Akissi, «Aux sources du théâtre ivoirien moderne : cas des premiers auteurs et leurs œuvres », Horizons/Théâtre [En ligne], 13 | 2018, mis en ligne le 01 janvier 2019, consulté le 28 juin 2022. URL : http://journals.openedition.org/ht/1166 ; DOI : https://doi.org/10.4000/ht.1166

\section{cc)}

La revue Horizons/Théâtre est mise à disposition selon les termes de la Licence Creative Commons Attribution - Pas d'Utilisation Commerciale - Pas de Modification 4.0 International. 


\section{FLORENCE KOUASSI AKISSI}

Florence Kouassi Aboua est enseignant-chercheur au département de Lettres modernes à I'Université de Cocody-Abidjan en Côte d'Ivoire. Elle a soutenu une thèse de doctorat en histoire littéraire ivoirienne à l'université de Cocody-Abidjan intitulée « La question du pouvoir politique dans la prose romanesque ivoirienne post-multipartisme 1990-2005 ». Elle a publié plusieurs articles dont " Le personnage de l'enfant soldat dans le roman ivoirien : cas de La Bible et le fusil, Allah n'est pas obligé et Le retour de l'enfant soldat », "Les naufragés de l'intelligence, fresque d'une société ivoirienne en crise. », " Historicisation et fictionnalisation du coup d'État ivoirien de 1999 dans le roman éponyme de Régina Yaou ». Elle est membre du Groupe d'étude et de recherche sur la littérature ivoirienne créé en 2005 et a participé dans ce cadre à la rédaction du Dictionnaire des romans ivoiriens publié chez l'Harmattan.

Mail: kouassiabflore@gmail.com

Résumé : Le théâtre occupe une place de choix dans l'univers littéraire ivoirien. En effet, en Côte d'lvoire, c'est par lui qu'est née la littérature ivoirienne. Mais I'histoire spécifique de ce genre est demeurée dans l'ombre des autres. II semble opportun de procéder à une immersion rétroactive, c'està-dire de rappeler la part que l'histoire littéraire peut prendre dans son avancée. D'où ce thème qui se veut un bref essai historiographique du théâtre ivoirien. Cette contribution ambitionne de revenir aux sources de

Abstract: The theater occupies a special place in the Ivory Coast literary universe. Indeed, in Ivory Coast, it is by him that was born the Ivory Coast literature. But the specific history of this kind lived in the shade of others. It seems convenient to proceed to a retroactive dumping, that is to call back the part which the literary history can take in its advance. From where this theme which aims to be a brief historiographical try of the Ivory Coast theater. This contribution aims la naissance de ce genre dans ce contexte qui fut le sien en tentant de répondre aux questions suivantes : Comment est-il né ? Pourquoi ? Quelles étaient les premières pièces? Comment furent-elles accueillies? Quelles furent les premières évolutions du théâtre ivoirien? Et comment a-t-il évolué jusqu'aux indépendances?

Mots-cLés : théâtre, histoire littéraire, Côte d'Ivoire, évolution.

to return to the sources of the birth of this genre in this context which was its own by trying to answer the following questions: How was it born? Why? What were the first pieces? How were they received? What were the first developments in Ivorian theater? And how did it evolve until Independence?

KEYworDs : theater, history literary person, Ivory Coast, evolution. 


\section{Aux sources du théâtre ivoirien moderne : cas des premiers auteurs et leurs œuvres}

\section{Introduction}

CET ARTICLE ENVISAGE DE REVISITER LES PRÉMICES DU THÉÂTRE en Côte d'Ivoire, dans une perspective historique et littéraire. Un tel rappel s'impose comme une nécessité vu l'importance de la place qu'occupe ce genre dans l'univers littéraire ivoirien. En effet, c'est par le théâtre que la littérature voit le jour en Côte d'Ivoire. Le droit d'aînesse lui confère dès lors une place privilégiée et des droits auxquels ne saurait déroger la littérature, la fiction, la création, dans son ensemble. La littérature ivoirienne est aujourd'hui riche d'auteurs et d'œuvres aux renommées internationales. Mais l'histoire spécifique de ce genre est souvent demeurée dans l'ombre des autres. En conséquence, par devoir de mémoire mais aussi par souci historiographique, il nous faut revenir sur les clés qui ont ouvert les portes de la littérature ivoirienne, en cerner le contexte de création, l'évolution. Ce qui pourrait être d'une quelconque utilité pour des études plus contemporaines en vue d'expliquer tel ou tel aspect de la littérature ivoirienne, dans son ensemble et de façon spécifique au théâtre. Au moment où le genre dramatique se présente comme la vitrine de la réflexion sur l'Afrique et de ses pratiques tous azimuts, il semble opportun de procéder à une immersion, c'est-à-dire de rappeler la part que l'histoire littéraire peut prendre dans son avancée.

Cet article ambitionne de revenir aux sources de la naissance du théâtre dans ce contexte qui fut le sien. Comment est-il né ? Quelles étaient les premières pièces ? Comment furent-elles accueillies ? Quelles furent les premières évolutions du théâtre ivoirien jusqu'aux indépendances ? Telles sont les principales questions qui nous guideront dans une perspective historiographique. L'histoire littéraire, qui dans la conception de Lanson, consiste à se fixer comme objectif de «tracer le tableau de la vie littéraire de la nation, l'histoire de la culture, et de l'activité de la foule qui lisait, aussi bien que les individus illustres qui écrivaient ${ }^{1} \gg$, constituera le socle méthodologique ma- 
jeur de ce travail dont l'objectif principal est de mieux appréhender aussi la logique particulière de ce genre en Côte d'Ivoire.

\section{De la naissance du théâtre ivoirien}

Au nombre des genres considérés a priori inhérents aux sociétés à tradition orale et existant déjà dans l'Afrique précoloniale, on note la poésie, le conte, le théâtre. Parler donc de l'existence du théâtre dans l'Afrique précoloniale revient à enfoncer une porte longtemps ouverte par la critique, tant la question a fait débat, et à trouver réponses après de convaincantes démonstrations. Mais pour la logique de l'argumentation, il nous faut revenir sur les prérequis qui relèvent maintenant de l'évidence. En effet, nombre de manifestations populaires, de rencontres populaires en Afrique précoloniale donnaient d'observer une forme de théâtre, différente dans le fond et dans la forme du théâtre européen formalisé comme le souligne Pairault : «Dans les cultures traditionnelles d'Afrique noire, le genre dramatique ne se présentait pas selon les formes canonisées par le même nom de théâtre ${ }^{2}$. Il n'empêche que, pour Barthelemy Kotchy, le théâtre fait partie du quotidien de l'Africain car «le moindre acte de la vie peut se muer en représentation dramatique ${ }^{3}$. »

Les séances de contes ou les cérémonies rituelles en Afrique sont de véritables spectacles offerts au public. Les battements de mains, les chants, les sons des tam-tams et les pas de danse rythmés sont entre autres des éléments plus ou moins théâtralisés. Il y a donc une théâtralité africaine traditionnelle et ancestrale. Ce critique ne dit pas autre chose quand il soutient que « le théâtre est l'expression de l'homme, de sa sensibilité, de ses émotions, de ses expériences ${ }^{4} \gg$. Ceci expliquerait en partie pourquoi nombre de littératures africaines sont nées soit par le conte soit le théâtre ou encore par la poésie.

C'est le genre dramatique qui donne naissance à la littérature ivoirienne, officiellement en 1933, avec Les villes de Bernard Dadié. De ce fait, le théâtre occupe une place de choix dans l'univers littéraire ivoirien. Mais la naissance de la première pièce ivoirienne demeure liée à deux éléments, à savoir l'influence du théâtre scolaire de la Gold Coast ${ }^{5}$ et les balbutiements du théâtre à l'EPS de Bingerville. Nous passerons ces deux moments en revue avant d'en arriver à la première pièce théâtrale.

Les élèves de la Gold Coast donnaient des spectacles à Grand-Bassam, ville côtière et important port de la Côte d'Ivoire coloniale jusque dans les années 1930. Ils improvisaient de courtes saynètes qu'ils déroulaient devant un public. Ce dernier y prenait une part active en exécutant des pas de 
danse, en entonnant avec les acteurs des chants. Cette communion entre les acteurs et le public se justifiait par le fait que les représentations se faisaient en langue locale, « l'apollonien », langue parlée par les habitants. C'est donc avec impatience que les populations attendaient les vacances scolaires pour retrouver ces troupes d'un autre genre car Bassam était la seule localité à bénéficier de ces représentations :

Les scènes n'étaient pas écrites. Les artistes improvisaient plutôt en paroles et gestes sur des thèmes de la vie courantes. Le répertoire comportait des saynètes de quelques minutes, parmi lesquelles Kodjo, le secrétaire et La tentation occupaient une place de choix 6 .

Gnaoulé Oupoh, dans l'ouvrage consacré à l'ensemble de la littérature ivoirienne $e^{7}$, rappelle que deux saynètes étaient prisées par les populations, Kodjo, le secrétaire et $\mathrm{La}$ tentation. Cette dernière pièce sera reprise par les élèves de Bingerville dont certains furent témoins des prestations comme le fait remarquer Amon d'Aby:

De nombreux élèves de l'EPS de Bingerville, comme Bernard Dadié, Vilasco Alexandre et bien d'autres sont originaires de Bassam. Ils ont vu jouer les élèves et la Gold Coast; c'est ce qui fait qu'on peut dire que nous avons eu des contacts avec le théâtre de la Gold Coast ${ }^{8}$.

Cependant, le cadre géographique de la naissance de la littérature ivoirienne demeure incontestablement l'EPS de Bingerville. En effet, en Côte d'Ivoire, il est aujourd'hui presque devenu une coutume de faire remonter les premiers balbutiements de la littérature moderne au sketch improvisé par deux élèves, à la rentrée scolaire de l'EPS de Bingerville en octobre 1932. Comme l'a confirmé François Joseph Amon D’Aby, les signes précurseurs de cette littérature s'inscrivent dans ce sketch, improvisé par deux élèves, qualifié de $\ll$ farce d'adolescents ${ }^{9} \gg$ qu'il décrit en ces termes :

Pour jouer la comédie, Edouard Aka Bilé avait pris l'initiative de passer un ceinturon sur la veste d'école. Je ne sais pas comment il s'est arrangé pour se procurer une chéchia rouge. Ce qui est sûr, c'est qu'il ressemblait parfaitement à un garde cercle. Armé d'une chicote, il poussait devant lui Robert Animan Amolin qui était vêtu d'une grande couverture pagne, comme un paysan. Edouard Aka Bilé le poussait en lui tenant le langage injurieux des gardes - cercles spécialisés dans les brimades; tous les élèves se sont mis à rire bruyamment ${ }^{10}$.

Cette saynète, brièvement présentée, mettait visiblement en cause le travail forcé qui était à l'époque le lot des indigènes colonisés, avec en point de mire 
le pouvoir colonial fait d'injustices et de violences. Alerté pas ce remue-ménage sans précédent,

Le directeur de l'école, Charles Béart qui n'en était qu'à sa deuxième année de séjour en colonie, a été pris de panique. Il résidait dans l'école, au-dessus des salles de classes et, de là il pouvait voir ce qui se passait dans la cour. Il est descendu rapidement, furieux, tout rouge de colère pour rechercher les causes de l'attroupement et punir sévèrement les auteurs de ce tumulte ${ }^{11}$.

Mais Charles Béart, qui a fini par comprendre l'enjeu de cet acte, eut une réaction intelligente pour son niveau de responsabilité dans l'administration coloniale. Ayant bien compris que ce pouvoir était tourné en dérision, en lieu et place d'une punition qui eût pu avoir pour effet de braquer les élèves, il fit construire un théâtre de verdure pour encourager la pratique théâtrale. Cela lui permit d'en assurer, in fine, le contrôle idéologique. De fait, les saynètes qui vont être produites par la suite porteront une tout autre thématique ne heurtant pas les intérêts coloniaux. Il en est ainsi de Le marchand de bangui ${ }^{12}$ de Yoman yoboué, Taylor, l'électricien ${ }^{13}$ avec Aka Bilé, La tentation ${ }^{14}$, Le sorcier et l'impô $t^{15}$ avec Alexandre Vilasco. Aucune de ces pièces ne sera écrite, il faut attendre 1933 pour voir écrire la toute première pièce théâtrale sous la plume de Bernard Dadié.

Avec Les villes, la première œuvre théâtrale, Bernard Dadié renoue avec la question du pouvoir politique dans la création dramatique et innove avec des personnages atypiques. La particularité de cette œuvre réside spécifiquement dans ces deux aspects : elle traite d'un problème politique et les personnages se révèlent atypiques. Les villes dénonce un problème lié à l'administration, donc au pouvoir colonial qui déplaçait chaque fois que bon lui semblait la capitale de la Côte d'Ivoire. Il y présente des villes personnifiées en train de se disputer le titre de capitale :

J'ai écrit une suite d'affrontements entre les capitales successives de la Côte d'Ivoire, Assinie, la première capitale ; Bassam, la deuxième, Bingerville, Abidjan... J'ai même ajouté Bouaké16.

Au cours de ce dialogue entre Assinie, Grand Bassam, Bingerville et Abidjan, les villes évoquent les transferts intempestifs de capitale avec son cortège de mouvements sociaux et économiques. L'ancienne capitale est délaissée parce qu'elle se vide de ses fonctionnaires européens et noirs au profit de la nouvelle élue. Il s'ensuit alors un vaste mouvement d'émigration des hommes d'une part, et d'autre part, des structures formelles ou infor- 
melles qu'impose le statut de capitale. Il y a là, comme le fait observer Nicole Vinciléoni, une critique politique sous-jacente :

La verve indépendante et satirique de l'adolescent se révélait déjà $[\ldots]$ puisqu'il montrait comment à peine en Côte d'Ivoire une ville s'accroissait-elle, prenaitelle de l'importance, qu'elle le faisait au détriment de la précédente, désormais abandonnée et qu'il y faisait sentir, sans rien qui pèse, ce qu'il avait de non ordonné dans la mise en valeur du territoire ${ }^{17}$.

Par ailleurs, cette œuvre bénéficie d'un ancrage réaliste, Grand-Bassam étant la première capitale de la Côte d'Ivoire. En effet, après leur installation en 1893, les Français firent de cette ville la capitale. Pendant près de sept ans, Grand Bassam était la capitale jusqu'à ce que la troisième épidémie de fièvre jaune en 1899 décime 45 Français sur les 60 présents dans la ville. Après ce drame en 1900, la capitale devint Bingerville.

Le succès de cette pièce a fait l'unanimité. La naissance du théâtre ivoirien révèle une création particulière, avec des personnages, des villes qui deviennent des êtres animés et crient leur désarroi, associée à une dénonciation politique certes discrète mais osée dans le contexte qui était le sien.

\section{L'Évolution jusqu'aux indépendances (continuité et rupture)}

L'évolution du théâtre ivoirien se produit dans deux espaces distincts : William-Ponty au Sénégal et la Côte d'Ivoire.

Après la naissance à l'EPS de Bingerville, les auteurs et acteurs de la vie théâtrale se retrouvent à William-Ponty au Sénégal, pour poursuivre leurs études. C'est la phase de William-Ponty. Deux œuvres ivoiriennes marquent ce passage : Assemien Dehylé, roi du Sanwi de Bernard Dadié inspiré de la légende et joué à la fête d'art scolaire et Les prétendants rivaux d'un collectif d'auteurs dont Amon d'Aby en est un co-auteur.

Dadié, comme à son habitude, avec Assemien Dehylé, roi du Sanwi, revient à la politique-fiction; dans son collimateur, la société traditionnelle, avec des thèmes sous-jacents comme l'initiation, le couronnement et le conseil des anciens, etc. Quant à la seconde pièce, Les prétendants rivaux, elle stigmatise l'avidité dans le mariage. Déjà improvisée à l'EPS, cette pièce sera écrite et représentée à Gorée et à la foire en France en 1937.

Ainsi, deux auteurs laissent leurs empreintes à William-Ponty avec de nombreux acteurs tels Robert Animan Amolin, Amon d'Aby, Dadié, Kokora Bitty, Mathieu Ekra, Alexandre Vilasco, Ignace Diplo, Jérôme Allo pour ne citer que ceux-là. 
De retour en Côte d'Ivoire, animés de la même verve concernant le théâtre, les auteurs vont poursuivre dans l'esprit de William-Ponty. Le théâtre ivoirien évoluera ainsi de 1938 à 1966. Puis à partir de cette date jusquà nos jours s'ouvrira une deuxième période du théâtre. La première période de 1938 à 1966, qui nous intéresse dans ce travail, comporte deux sous-ensembles distincts. Le premier s'étend sur sept ans de 1938 à 1943 et le second sur cinq ans de 1953 à 1958.

La période de 1938 à 1943 est dominée par le Théâtre Indigène de Côte d'Ivoire (TICI), créé en 1938 par Amon d'Aby et Germain Coffi Gadeau qui en seront les principaux auteurs. Le premier produit trois pièces intitulées L'entrevue de Bondoukou en 1939, Boussatié en 1940 et La mort de la princesse Alloua en 1941. Coffi Gadeau, quant à lui, en donne six : Kondé Yao en 1939, Nos femmes en 1940, Mon mari, Les recrutés de monsieur Maurice, Les anciens combattants en 1942 et Le mariage de Sogona en 1943.

Le challenge que relève le TICI est qu'il parvient à faire du théâtre non plus uniquement le fait exclusif des élèves, mais l'affaire de toute la population. Le mérite du TICI est donc d'avoir élargi le public du théâtre ivoirien.

Coffi Gadeau se présente come la figure de proue de cette période à cause de la censure d'une de ses œuvres. En effet, l'administration coloniale a jugé nécessaire d'interdire la pièce de Gadeau, Les recrutés de monsieur Maurice, qui soulève le problème du travail forcé lors du sketch improvisé dix ans plus tôt par les élèves de l'EPS de Bingerville. Pour rappel, ce fut la même thématique. Charles Béart avait alors subtilement étouffé les velléités.

Le second moment - 1953 à 1958 - est marqué par les centres culturels. Conçue comme des appuis logistiques importants pour les associations culturelles et sportives de jeunes, la création des centres culturels en AOF en 1953 avait pour mission de contrôler la vie culturelle dans les colonies. La décision de la création des centres culturels fut imposée dans toute l'AOF avec en sus un journal de liaison dénommé Trait d'union. L'essaimage des centres culturels fut immédiat. En Côte d'Ivoire, les premières villes qui en furent dotées sont Bouaké, Man, Gagnoa et Dimbokro. Pour les animer et les relier, une revue de liaison, Trait d'Union ${ }^{18}$, fut éditée sur des fonds du Gouvernement général de l'AOF.

Mais c'est le Centre Culturel Folklorique de Côte d'Ivoire (CCFCI), créé en Côte d'Ivoire avant le boom des centres culturels, qui animera cette période avec sa section consacrée au théâtre. Le trio célèbre des anciens pontins produit des œuvres en adéquation avec la colonisation ; un théâtre dit « pragmatique et social $^{19}$ » parce que convenable à la colonisation. 
Dans ce registre, Bernard Dadié produit cinq pièces : Min Adjao en 1955, Serment d'amour en 1955, Situation difficile en 1955, Les enfants en 1956 et Siddi maitre escroc en 1957. On en enregistre deux de Coffi Gadeau : Yaou $N^{\prime} D a$ en 1954 et Adjo Bla en 1955. Amon d'Aby est l'auteur de quatre pièces de théâtre : Kwao Adjoba en 1955, Entraves en 1956, La couronne aux enchères et La sorcière en 1957.

\section{Conclusion}

Le théâtre ivoirien a suivi l'itinéraire de l'école coloniale ; en effet, de Bassam où certains écoliers furent témoins des troupes scolaires de la Gold Coast et leurs jeux à l'EPS de Bingerville où il est né à William Ponty au Sénégal pour la confirmation aofienne et internationale puis le retour en Côte d'Ivoire. Un parcours circulaire, typique du trajet des intellectuels de cette période qui quittent leur village après avoir fait leurs premiers pas à l'école du village, pour se retrouver à l'EPS pour ceux qui passent brillamment ce cap de l'école régionale. Après l'EPS, c'est William-Ponty qui les accueille. Puis c'est le retour au pays qui peut être différé par un détour de formation additionnel en France.

Influence produite à Bassam, certainement école du village ou régionale, c'est l'EPS qui marque la naissance du théâtre en Côte d'Ivoire. L'affirmation et la confirmation du genre s'opèrent au Sénégal puis au retour en Côte d'Ivoire, avec des résultats plus ou moins probants, avec le TICI puis le CCFCI. La période jusqu'à l'indépendance est, dans l'ensemble, marquée par des pièces qui apportent de l'eau au moulin des préjugés de la colonisation. La thématique demeure en faveur de la colonisation. Quand ce ne fut pas le cas, deux types d'actions ont été observés chez le colon : la récupération stratégique ou la censure brute.

Le théâtre ivoirien a dérangé l'administration coloniale, par son réalisme et sa dénonciation. Quand, dès ses balbutiements, Charles Béart avait flairé le danger que pouvait représenter le théâtre dans la prise de conscience des populations colonisées, il a su de manière stratégique, sans éveiller les soupçons, en prendre le contrôle idéologique. Il organisait lui-même les auteurs, les encadrait, les cachait et donc tout naturellement les orientait. La réaction fut plus brutale dix ans plus tard, en 1942, avec l'interdiction pure et simple de la pièce de Germain Coffi Gadeau. La période qui ouverte avec l'indépendance est sans doute plus acerbe dans la critique car « il faut que notre production théâtrale reflète la vie africaine avec réalisme ${ }^{20} \gg$. 


\section{Notes}

1. Gustave Lanson : «Programme d'études sur l'histoire provinciale de la vie littéraire en France » in Études d'bistoire littéraire, Paris Champion, 1929.

2. Claude Pairault, "Où trouver le théâtre " in Actes du colloque sur le théâtre négro-africain, Paris, Présence Africaine, 1970, p. 16.

3. Barthélemy Kotchy, Éléments culturels et formes de représentations en Afrique. L'exemple de la Côte d'Ivoire, thèse d'État ès lettres, Université de Paris VII-Vincennes, 1983, vol. 1 p. 167-168.

4. Bakary Traoré, Le théâtre négro-africain et ses fonctions sociales, Paris, Présence Africaine, 1958, p. 11.

5. Gold Coast est l'actuel Ghana, ancienne colonie sous domination britannique.

6. François Amon d'Aby, Le théâtre en Côte d'Ivoire, des origines à nos jours, Abidjan, CEDA, 1988, p. 16.

7. On y lira les résumés de ces deux pièces.

8. Oupoh Gnaoulé, « Entretien avec F.-J. Amon d'Aby, dramaturge ivoirien », Abidjancocody, 18-20 avril 1988, p. 40, texte dactylographié de 125 p., inédits.

9. François Amon d'Aby, Le théâtre en Côte d'Ivoire, des origines à nos jours, op. cit., p. 9.

10. Gnaoulé Oupoh, La littérature ivoirienne, Paris-Abidjan, Karthala-CEDA, 2000, p. 29.

11. Ibid., p. 30.

12. Yoman yoboué était un personnage jouant le rôle du vendeur de bangui, vin extrait du cœur du palmier. Ce vin est de consommation très courante en Côte d'Ivoire où il est vendu un peu partout au coin des rues. Des élèves, représentant les différentes couches sociales de la population, s'approchaient du personnage comique et discutaient autour du bangui.

13. C'est l'imitation sur scène du comportement d'un électricien d'origine sierra léonaise nommé Taylor, qui exerçait ses talents à Bingerville. Quand on faisait appel à ses services pour une panne d'électricité, il ne mettait jamais la main à la pâte. Taylor restait toujours au pied du poteau et faisait monter quelqu'un à sa place et lui donnait des instructions plus ou moins heureuses.

14. Elle présente une classe de catéchisme. C'était au début de l'évangélisation et les enfants studieux écoutaient avec attention la leçon du jeune catéchiste noir. Mais un accordéon drainant une troupe de bambins perturbe la sérénité de la classe : les catéchumènes nécoutent plus. Malgré les multiples signes de croix pour ne pas succomber à la tentation, les enfants ne résistent pas, sortent et se mêlent à la danse. Le pauvre catéchiste, tout seul, finit par rejoindre à son tour la bande joyeuse.

15. Cette saynète met en scène un sorcier appelé au chevet d'un malade; par de nombreuses gestes et contorsions, il faisait semblant d'extraire le mal sous la forme de fléchettes ou de petits cailloux.

16. Richard Bonneau, "Dadié écrivain ivoirien » in Entente africaine, n 10, Abidjan, juillet 1972, p. 52.

17. Vinciléoni Nicole, Présentation de Assémien Déhylé roi du Sanwi, Abidjan, CEDA, 1979, p. 111

18. L'administration attacha beaucoup de soins à sa réalisation et à sa diffusion. De 1953 à 1957, avec 19 numéros qui constituèrent un lien entre les différents centres, il contri- 
Aux sources du théâtre ivoirien moderne : cas des premiers auteurs et leurs œuvres

bua à développer le sentiment d'appartenance à l'AOF. La revue était principalement rédigée par des intellectuels africains comme Ahmadou Hampaté Bâ, Boubou Hama, etc. et comportait diverses rubriques.

19. François Amon d'Aby, Le théâtre en Côte d'Ivoire, des origines à nos jours, Abidjan, CEDA, 1988, p. 11.

20. Bakary Traoré, Le théâtre négro-africain et ses fonctions sociales, Paris, Présence Africaine, 1958, p. 143. 